\title{
Virtual Wave Concept for thermographic parameter estimation using modulated laser excitation
}

\author{
by H. Plasser*, G. Mayr*, G. Thummerer*, G. Hendorfer*, P. Burgholzer** \\ * University of Applied Sciences Upper Austria, Josef Ressel Centre for Thermal NDE of Composites, Franz- \\ Fritsch-Straße 11, 4600,Wels, Austria, holger.plasser@fh-wels.at \\ ${ }^{* *}$ RECENDT - Research Center for Non Destructive Testing GmbH, Altenberger Straße 69, 4040, Linz, Austria, \\ peter.burgholzer@recendt.at
}

\section{Abstract}

In this work, the Virtual Wave Concept is applied on measurement data from laser-excited thermography. Therefore, a virtual temperature signal is calculated by applying a local transformation to the temperature data from the IR camera. The propagation of the virtual temperature can be described by the wave equation, whereby for the parameter estimation ultrasonic evaluation methods can be used, e.g. pulse-echo method for time-of-flight measurements. The timeof-flight determined from the virtual temperature is directly related to the thermal diffusion time. The determination of the thermal diffusion time with the Virtual Wave Concept allows a thickness estimation of a step wedge sample as well as a porosity estimation, using effective medium theories, of different test coupons.

\section{Introduction}

An unavoidable defect in carbon fiber reinforced plastics (CFRP), caused by the formation of voids during the manufacturing process, is porosity. Particularly, the autoclave molding of pre-impregnated fibers (prepreg fabrics or unidirectional tapes) is a critical step in manufacturing. Enclosing air during the lay-up and insufficient resin hydrostatic pressure results to keep any moisture or volatiles dissolved in the resin until gellation occurs [1]. This is the reason for the incurrence of voids. The presence of porosity has noticeable effects on mechanical properties since voids weaken the epoxy matrix. Especially, the matrix-dominated material properties such as the transverse tensile strength and the interlaminar shear strength decrease with increasing porosity [2, 3]. For safety relevant CFRP components and structures, the characterization of porosity is indispensable.

The aim of this work is the quantitative non-destructive evaluation of porosity with laser-excited thermography using the Virtual Wave Concept (VWC). This is demonstrated on multidirectional CFRP for the first time. In the first step, ultrasonic time-of-flight techniques are used to determine the position of the backwall of different steps on a CFRP step wedge. Based on these results the thermal diffusion time and hence the effective thermal diffusivity are determinable. In a second step, an effective-medium-model based approach is shown for the porosity estimation on calibrated porosity coupons (5-, 10- and 20-plies).

\section{Method: Virtual Wave Concept}

In this work, the quantitative evaluation of porosity in carbon fiber reinforced plastics with laser-excited thermography data, is based on the VWC [4]. Therefore, a virtual temperature signal $T_{\text {virt }}$ is calculated by applying a local transformation at the position $\mathbf{r}$ to the measured surface temperature data $T$. This transformation is a linear inverse problem and can be formulated as a Fredholm integral of the first kind

$$
\int_{-\infty}^{\infty} K\left(t, t^{\prime}\right) T_{\mathrm{virt}}\left(\mathbf{r}, t^{\prime}\right) d t^{\prime}=T(\mathbf{r}, t)
$$

where the right-hand side $T$ and the kernel $K$ are in principal known functions, while $T_{\text {virt }}$ is the unknown virtual temperature field. The time is given by $t$, and the virtual time is denoted as t'. The aim is to find the virtual wave field $T_{\text {virt }}$ from the measured temperature field $T$ and the corresponding model $K$. The kernel $K$ describes the linkage between the heat conduction equation and the wave equation

$$
K\left(t, t^{\prime}\right) \equiv \frac{c}{\sqrt{\pi \alpha t}} \exp \left(-\frac{c^{2} t^{\prime 2}}{4 \alpha t}\right) \text { for } t>0,
$$

where $\alpha$ is the thermal diffusivity and $c$ the virtual speed of sound. Since thermography, data is discrete in time and space domain we discretize Eq. 1:

$$
\mathbf{T}=\mathbf{K} \mathbf{T}_{\text {virt }}
$$


Herein, $\mathbf{T}$ and $\mathbf{T}_{\text {virt }}$ are the vectors of the measured temperature signal and the virtual wave signal. Since the problem is severely ill-posed, the truncated singular value decomposition (T-SVD) and the alternating direction method of multipliers (ADMM) [5] are used as regularization techniques. Prior information, such as positivity and sparsity of the virtual wave field, is included in the ADMM procedure to improve the reconstruction quality (Fig. 1(a)). The propagation of the virtual temperature can be described by the wave equation, whereby for parameter estimation well-established ultrasonic methods can be used, e.g. pulse-echo method for time-of-flight measurements [6]. In Fig. 1(b) the A-Scan representation of the analytical virtual wave and the regularized solution with the classical truncated singular value decomposition and ADMM is shown.
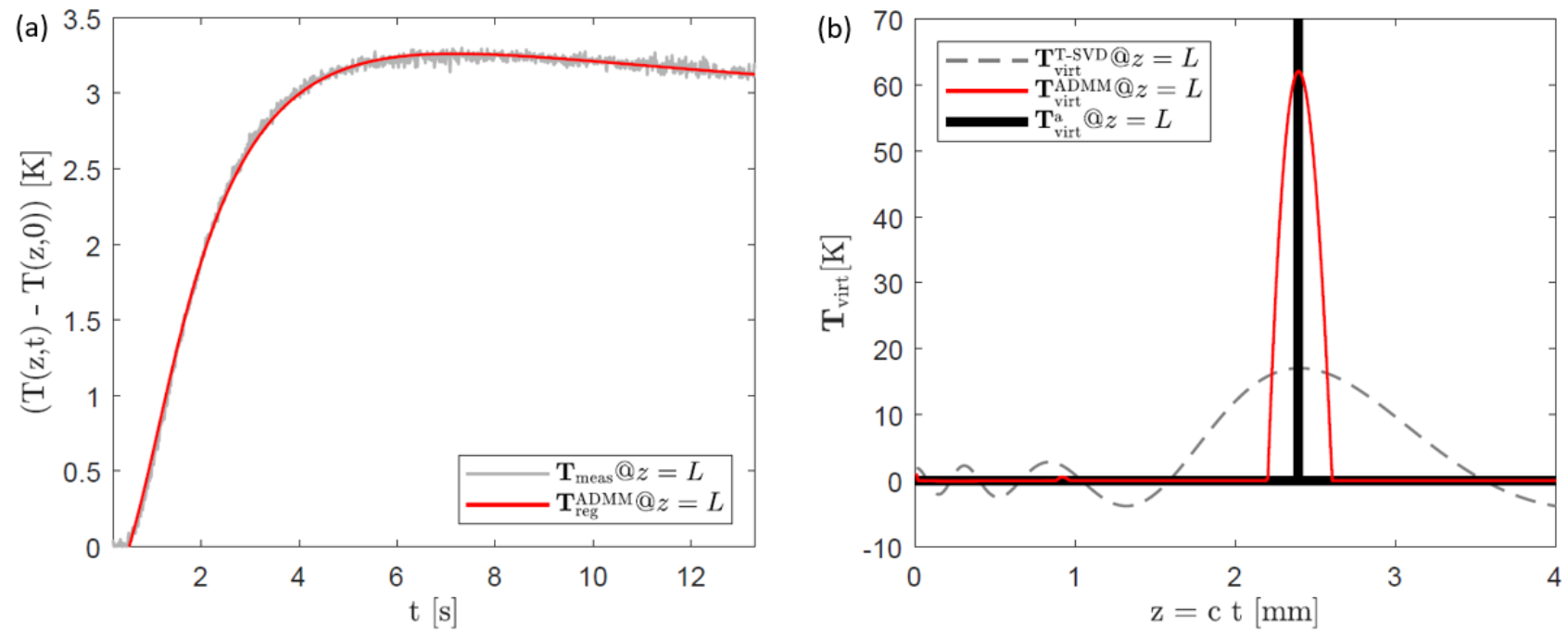

Fig. 1: Comparison between measured and regularized transmission mode temperature signal (a), virtual A-Scan comparison between ideal virtual wave and different regularization techniques (b)

The time-of-flight determined from the back-wall echo of the virtual temperature signal is directly related to the thermal diffusion time $t_{D}=L^{2} / \alpha$. Therefore, if the specimen thickness $L$ is known the effective thermal diffusivity can be calculated and vice versa.

\section{Results and Conclusion}

The measurement of the thermal diffusion time with the VWC allows a thickness estimation of a CFRP step wedge sample as well as a porosity estimation of different CFRP test coupons. The measurement uncertainty of the VWC method was determined from the measurement data of the step wedge. Furthermore, effective medium theories are used to derive the porosity from the estimated thermal diffusivity on a range of different calibrated CFRP specimens. All thermography results are validated with X-ray computed tomography. The main advantage of the VWC is the possibility to use the same data algorithm for laser-excited thermography measurements in reflection as well as in transmission configuration for parameter estimation.

\section{Acknowledgements}

The financial support by the Austrian Federal Ministry of Science, Research and Economy and the National Foundation for Research, Technology and Development is gratefully acknowledged.

\section{REFERENCES}

[1] F.C. Campbell, Structural composite materials (ASM Internat, Materials Park, Ohio, 2010).

[2] P. Olivier, J.P. Cottu, B. Ferret, Composites (1995) doi:10.1016/0010-4361(95)96808-J.

[3] M.L. Costa, S.F.M.d. Almeida, M.C. Rezende, Composites Science and Technology (2001) doi:10.1016/S02663538(01)00157-9.

[4] P. Burgholzer, M. Thor, J. Gruber, G. Mayr, Journal of Applied Physics (2017) doi:10.1063/1.4978010.

[5] R.C. Aster, B. Borchers, C.H. Thurber, Parameter estimation and inverse problems (Elsevier, Amsterdam, Netherlands, 2019).

[6] J. Krautkrämer, H. Krautkrämer, Ultrasonic Testing of Materials (Springer Berlin Heidelberg, Berlin, Heidelberg, 1990). 